Accepted for publication in "Cognitive Therapy and Research". Note: This is an uncorrected version of an author's manuscript accepted for publication. Copyediting, typesetting, and review of the resulting proofs will be undertaken on this manuscript before final publication. During production and pre-press, errors may be discovered that could affect the content.

\title{
Hopelessness: A network analysis
}

\author{
Igor Marchetti ${ }^{1,2}$ \\ ${ }^{1}$ Department of Experimental-Clinical and Health Psychology, Ghent University, Belgium \\ ${ }^{2}$ Department of Health Sciences, Psychology Section, University of Florence, Italy
}

Corresponding author: Igor Marchetti, E-mail: igor.marchetti@ugent.be

\section{Acknowledgements}

Igor Marchetti is a postdoctoral research fellow, supported by the Research Foundation Flanders (FWO14/PDO/115). The author has no competing interests in this research. The author wants to thank dr. Ernst H. W. Koster for his thoughtful and constructive comments on a previous version of this article. 


\begin{abstract}
Hopelessness is a complex phenomenon with important clinical consequences, such as depression and suicidality. Despite its major impact on mental health, little is known about the structure of hopelessness. In this study, hopelessness was investigated in a large community sample $(n=1985)$, recruited to be highly representative of general population in Germany. In the context of network analysis, state-of-the-art techniques were adopted (i) to investigate which thoughts and beliefs (nodes) are the most central ones and (ii) to shed light on the specific associations (edges) among them. Stability and accuracy were also checked to ensure trustworthiness of the findings. The analyses revealed that expecting more negative than positive future events and having important goals blocked along with feelings of giving-up were the most central elements of hopelessness. Moreover, being unable to imagine the future and perceiving it as vague and uncertain were both coupled with anticipating a dark future. Theoretical and clinical consequences of this study were discussed.
\end{abstract}

Keywords: hopelessness, Beck Hopelessness Scale, network analysis 


\section{Introduction}

Hopelessness is defined as the expectation that negative events will occur and/or positive events will not occur, along with the belief that the person can do nothing to change this gloomy scenario (Abramson, Alloy, \& Metalsky, 1989). Such a negative attitude toward the future is often reported in major psychopathological conditions, namely depression and schizophrenia (Beck, Steer, Beck, \& Newman, 1993; Lysaker, Davis, \& Hunter, 2004), but it is also present at clinical levels in the general population (i.e., $10 \%$; Haatainen, Tanskanen, Kylma, Honkalampi, et al., 2003). Furthermore, high levels of hopelessness have been concurrently and prospectively associated with clinical outcomes, such as depression (Mac

Giollabhui et al., 2018; Marchetti, Loeys, Alloy, \& Koster, 2016) and suicidality (Franklin et al., 2017). In sum, hopelessness is a phenomenon that deserves great clinical and research attention.

Despite its importance, however, key aspects of hopelessness are still opaque. For instance, hopelessness is characterized by a variety of components, among which biased future thinking (i.e., reduced ability to generate positive future events; Roepke \& Seligman, 2016; “I can't see any future for myself or the rest of the human race", Ratcliffe, 2015, p. 112), helplessness (i.e., feelings of inability to bring about any significant change; Seligman, 1975; "I have a feeling of pointlessness and inevitability of outcome so feel powerless to make changes"; Ratcliffe 2015, p. 112), and blocked goal-processing (i.e., beliefs that the pursue of meaningful goals is impeded along with feelings of giving-up; Hadley \& MacLeod, 2010; Melges \& Bowlby, 1969, "I'll never get what I want and need -and, that is really horrible"; Crawford \& Ellis, 1989, p 13). In spite of this complexity, however, a thorough examination of the inner structure of hopelessness and the interaction among its constituent elements is still lacking. 
Traditional theorizing and standard statistical approaches primarily view psychological phenomena as unobservable factors that generate observable indicators (i.e., latent factor approach). A key assumption of this approach is that indicators have no causal influence on each other, but they simply reflect the underlying latent factor (Schmittmann et al., 2011). It follows that, within the latent factor approach, imagining a negative future, having no feelings of control, and expecting to miss important goals are not supposed to interact with one another, but they are considered as static manifestations of hopelessness. Hence, this view cannot fully capture and articulate the ongoing process among the different components of complex phenomena like hopelessness (Dalege et al., 2016; Schmittmann et al., 2011).

Alternative to the latent factor approach, a new way to conceptualize psychological phenomena has recently been proposed, namely network approach (Borsboom \& Cramer, 2013). According to this perspective, psychological constructs (i.e., hopelessness) do not necessarily stem from latent factors, but they may emerge from the mutual interactions among their observable indicators (i.e., questionnaire items; Briganti, Braun, Fried, \& Linkowski, 2018; Costantini \& Perugini, 2018; Dalege et al., 2016). For example, the belief that one's own behavior does not bring about any positive consequence could lead the person to believe that his/her future is dark, which, in turn, could elicit feelings of giving-up. Over time, the reiteration of this (simplified) sequence of thoughts could facilitate the development of a densely connected network, where most of the beliefs reinforce one another and eventually lead to generalized hopelessness (i.e., Costantini \& Perugini, 2018; Dalege et al., 2016). In sum, network analysis approaches psychological constructs from a different perspective as compared to traditional models, in that it primarily focuses on the single elements of the construct (i.e., nodes) and how they relate to one another (i.e., edges), putatively in a causal fashion (Borsboom \& Cramer, 2013; Dalege et al., 2016). By doing so, 
such a perspective offers new opportunities to shed light on the structure and functioning of important psychological phenomena (Schmittmann et al., 2011). In particular, network analysis can help identify (i) which nodes are the most central in the structure of network (see below for more details) and (ii) which edges between nodes function as the main pathways of the network. For all these reasons, network analysis is highly valuable approach both for describing psychological constructs and generating testable hypotheses for further research (Borsboom \& Cramer, 2013; Costantini \& Perugini, 2018).

The main goal of this study is to preliminarily investigate the phenomenon of hopelessness by means of network analysis. To do so, I will closely examine the Beck Hopelessness Scale (BHS; Beck, Weissman, Lester, \& Trexler, 1974), which is as wellestablished measure for hopelessness in adults and is routinely used for assessing this construct in both clinical and non-clinical samples (Beck et al., 1993; Haatainen, Tanskanen, Kylma, Antikainen, et al., 2003; Haatainen, Tanskanen, Kylma, Honkalampi, et al., 2003). An appropriate network model on the BHS items will be estimated. Then, the strength index will be used to identify the most central nodes, while the predictability index will quantify how much each node is accounted for by the neighboring nodes. Finally, with the important aim to obtain reliable and trustworthy results, this study will be carried out in a large community sample (i.e., 2000 individuals), specifically recruited to be highly representative of the general population in Germany (Krampen, 1994).

\section{Method}

\section{Participants}

The initial sample consisted of 2051 individuals. Given that the presence of missing data may alter the structure of the network (Borsboom et al., 2017), sixty-six individuals were excluded. The final sample consisted of 1985 individuals (46.15\% males; age $45.2 \pm 17.8$ 
years old; range $=18-90$ ). Different age groups were included, ranging from young adults to elderly $(18-25=12.7 \% ; 25-40=33.5 \% ; 41-64=35.2 \% ; 65-90=18.6 \%)$. Moreover, $51 \%$ of the individuals reported having either a full-time or part-time job, while $49 \%$ reported not having a paid job (6.6\% students, $21.4 \%$ retired, $1.8 \%$ unemployed; $18.2 \%$ homemakers; $1 \%$ other).

Importantly, this sample was recruited to be highly representative of the general population with the purpose to standardize the German version of the Beck Hopelessness Scale (Krampen, 1994). Recruitment was carried out in line with the German Market Research Institute and consisted of 420 sample points, stratified random sampling method, random route, and random choice of target persons in the household. Data are publicly available from the Leibniz Institute for Psychology Information (Krampen, 2004).

\section{Measure}

Beck Hopelessness Scale (BHS; Beck et al., 1974). The BHS consists of 20 statements, with 11 negatively worded items (i.e., "My future seems dark to me") and 9 positively worded items (i.e., "I look forward to the future with hope and enthusiasm"). Previous studies did not identify a stable factor structure for this instrument, with up to five factors being reported in the literature (Hanna et al., 2011; Kliem, Lohmann, Mößle, \& Brähler, 2018; Zhang, Jia, Hu, Qiu, \& Liu , 2015). Although the original BHS items were rated on dichotomous items ("true" and "false"), the German version of the instrument used a 6-point Likert scale, ranging from 1 ("strongly agree") to 6 (“strongly disagree"). Participants were required to evaluate their degree of agreement with each item. It is worth mentioning that the use of polytomous items for the BHS has been subsequently adopted in several studies (Iliceto \& Fino, 2015; Marshall et al., 1992; Steed, 2001). Also note that positive items were reversed and, for sake of clarity, all the items were recoded, in such a way that 
higher values indicate greater levels of hopelessness.

\section{Statistical Analysis}

First, mean, standard deviation, kurtosis, skewness, and polychoric correlations of all the BHS items were inspected. Further, the informativeness of each variables was evaluated by means of standard deviation (Mullarkey, Marchetti, \& Beevers, 2018) and possible items redundancy was checked. In accordance with Jones (2018), two items (i.e., "A" and "B") were deemed to measure the same phenomenon (i.e., redundancy), if the polychoric correlations between $\mathrm{A}$ with the rest of the items and $\mathrm{B}$ and the rest of the items were statistically different less than $25 \%$ of the cases. The item redundancy test was carried out with the R package networktools 1.1.1 (Jones, 2018).

Second, an EBIC graphical LASSO network model with all the items was estimated, in line with current guidelines (Epskamp \& Fried, 2018). In detail, the association between every pair of variables was computed with polychoric correlation, after controlling for all the other variables included in the network. Further, in order to shrink small correlations to exact zero, the least absolute shrinkage and selection operator (LASSO) was used and the related tuning parameter was chosen with the Extended Bayesian Information Criterion (EBIC). By doing so, all the non-zero correlations are conditionally dependent (i.e., not spuriously due to any other variable of the network) and the network is sparser and easier to interpret. When dealing with binary variables, an Ising model was estimated (van Borkulo et al., 2014). Similarly to the EBIC graphical LASSO network estimation, the Ising model can be conceived as a series of pairwise associations between dichotomous variables, after controlling of all the other variables included in the network (for more details, see van Borkulo et al., 2014). In the context of network analysis, every variable is defined node and the link between two nodes is defined edge. Blue edges indicate positive associations, while red edges indicate negative ones. More saturated ad thicker edges signify stronger 
associations between two nodes. For the estimation and visualization of the network, the Rpackages qgraph 1.5 and bootnet 1.0.1 were used (Epskamp, Borsboom, \& Fried, 2018; Epskamp, Cramer, Waldorp, Schmittmann, \& Borsboom, 2012).

Third, local network properties were evaluated with two metrics, such as strength and predictability, using the R-packages qgraph 1.5 and mgm 1.2-2 (Epskamp et al., 2018; Haslbeck \& Waldorp, 2018). Strength is defined as the sum of the absolute weights of the edge connecting the node to all the other nodes (Valente, 2012), while predictability quantifies how well a certain node is predicted by all its neighboring nodes (Hanslbeck \& Waldorp, 2018). Hence, the predictability index ranges from 0 to 1 and represents the amount of variance of certain node accounted for by all the related nodes.

Fourth, to ensure robustness of network models (Epskamp et al., 2018), accuracy and stability of the network model were investigated with a two-fold approach: a) centrality stability and bootstrapped difference test for the centrality index; b) edge accuracy and bootstrapped difference test for edges. Strength was deemed stable if the correlation stability coefficient (i.e., CS-coefficient), was above 0.25 , but preferentially above 0.5 . Then, the difference between two strength indices was considered significant if 1000-bootstrap 95\% non-parametric confidence intervals (CIs) did not contain zero. Similarly, edge accuracy was estimated with $95 \%$ bootstrap CIs, with larger CIs suggesting reduced precision in the estimation of the edges and narrower CIs implying a more trustworthy network. Significant differences among edges were estimated as CIs, with two edges being statistically different if zero was not included.

\section{Results}

\section{Descriptive statistics}

Mean, standard deviation, skewness, kurtosis, and polychoric correlations of all the BHS items are reported in Table S1 and Table S2. Moreover, on average, individuals tended 
to disagree with statements related to hopelessness (i.e., below the midpoint of $3 ; M=2.76 \pm$ $\left.0.39, t_{(19)}=2.72, p<.02\right)$.

\section{Network estimation and local network properties}

Preliminarily, item informativeness (i.e., standard deviation of the item) and item redundancy were checked. No item was found to be poorly informative (i.e., 2.5 SD below the mean level of informativess, $M_{S D}=1.29 \pm 0.09$ ) and no item was found to be redundant with any other item (i.e., less than $25 \%$ of statistically different correlations). Hence all the items were included in the analyses.

The network of beliefs about hopelessness is shown in Figure 1. Several points are noteworthy. First, specific nodes were highly connected with the rest of the network, such as node \#17 (i.e., "It's very unlikely that I will get any real satisfaction in the future”) and \#15 (i.e., "I [don't] have great faith in the future"), while others appeared to be somewhat marginal, such as node \#5 (i.e., "I [don't] have enough time to accomplish the things I most want to do").

Second, the inspection of the local network structure revealed that two items had the highest centrality index and were statically more central than the other nodes, namely \#19 (i.e., "I can [not] look forward to more good times than bad times", strength index $=1.20$ ) and \#16 (i.e., "I never get what I want, so it's foolish to want anything", strength index $=1.20$ ) (Figure 2 and Figure 3). It is worth stressing that the strength index in this sample was particularly robust and trustworthy $(\mathrm{CS}$-coefficient $=0.75)$, in that dropping up to $75 \%$ of the sample would lead to extremely similar results (Epskamp et al., 2018).

Third, the predictability index showed that on average about $36 \%$ of each node's variance could potentially be accounted for by the neighboring nodes $\left(M_{\text {predictability }}=0.36 \pm\right.$ 0.13). However, while node \#5 was poorly explained (predictability = 0.10), node \#16 was 
substantially accounted for (predictability $=0.56$ ). It is worth stressing that, on average, the majority of variance (i.e., 64\%) in the network was unexplained.

Fourth, in this sample strength and predictability were not related with item variability $\left(r_{s}=-0.29[-0.65 ; 0.16]\right.$ and $r_{s}=-0.04[-0.47 ; 0.41]$, respectively), but negatively correlated with the item mean levels $\left(r_{s}=-0.57[-0.81 ;-0.17]\right.$ and $r_{s}=-0.58[-0.81 ;-0.18]$, respectively). In other words, the most central and, in turn, the most predictable nodes were likely to be the items with the lowest mean, such as node \# $16(M=2.23$, "I never get what I want, so it's foolish to want anything"), node \#17 ( $M=2.26$, "It's very unlikely that I will get any real satisfaction in the future"), and node \# $9(M=2.28$, "I just can't get the breaks, and there' is no reason to believe I will in the future").

\section{Accuracy and edge comparisons}

The edge accuracy test suggested that the precision of the 190 edges was excellent (Figure S1) and, consequently, the network model was deemed as accurate. The analysis revealed that the edges among node \#16 (i.e., "I never get what I want, so it's foolish to want anything"), \#17 (i.e., "It's very unlikely that I will get any real satisfaction in the future"), and \#20 (i.e., “There's no use in really trying to get something I want because I probably won't get it") were statistically different from the vast majority of the other edges. Similarly, the edges among node \#4 (i.e., "I can't image what my life would be like in 10 years"), \#7 (i.e., "My future seems dark to me"), and \#18 (i.e., "The future seems vague and uncertain to me") were statically stronger than the majority of the network edges. Then, node \#12 (i.e., "I don't expect to get what I really want") and \#14 (i.e., "Things just won't work out the way I want them to") were among the strongest edges of the network. Finally, node \#15 (i.e., “ $I$ [don't] have great faith in the future") and \#19 (i.e., "I can [not] look forward to more good times than bad times") emerged as statistically different from most of the other edges (Figure S2).

\section{Covariating age, gender, and employment status}


Previous evidence showed that age, gender, and employment status might influence hopelessness (Greene, 1981; Haatainen, Tanskanen, Kylma, Antikainen, et al., 2003; Haatainen, Tanskanen, Kylma, Honkalampi, et al., 2003). Hence, in line with Dalege, Borsboom, van Harreveld, and van der Maas (2017), the network model and the local structure indexes were re-estimated, after controlling for age, gender, and employment status. As compared with the original network, an almost identical network was obtained with respect to edges magnitude $(r=0.91[0.89 ; 0.92])$, strength $\left(r_{s}=0.86[0.67 ; 0.94]\right)$, and predictability $\left(\mathrm{r}_{\mathrm{s}}=0.99[0.98 ; 0.99]\right)($ Figure $\mathrm{S} 3)$.

\section{Estimating the network on dichotomized items}

In order to check the robustness of the presented results and make them informative for future studies using the original dichotomous BHS items (Beck et al., 1974), the polytomous items were dichotomized by the midpoint (i.e., 3). Then, an Ising model with dichotomized items was estimated (Figure S4). Importantly, highly similar results were obtained in terms of edge values $(r=0.88[0.86,0.90])$ and strength $\left(r_{s}=0.87[0.69,0.94]\right.$, and moderately similar in terms of predictability $\left(r_{\mathrm{s}}=0.55[0.14,0.80]\right)$.

\section{Discussion}

Hopelessness is a complex phenomenon that is reliably associated with important clinical outcomes, such as depression and suicidality. Despite its role in mental health, however, little known is about the structure of its constituent elements and how these components interact with one another. Complementing previous studies relying on the latent factor approach, network analysis was adopted to improve our understanding of this phenomenon.

Several findings are worth commenting. First, the reported analysis revealed that individuals from the general population tend to view their future in a hopeful way rather than 
in a hopeless manner. This is in line with previous literature suggesting that, overall, individuals are characterized by significant levels of optimism (Fischer \& Chalmers, 2008; Peterson, 2000).

Second, network structure analysis showed that being certain that important goals will not be reached along with feelings of giving up on wanting (node \#16) was one of the two most central items in the network. Interestingly, this node was strongly associated with the expectation that no real satisfaction will probably be obtained (node \#17) and the feelings of stop trying to achieve the set goals (node \#20). Node \#16 was also substantially related to feelings of not having lucky opportunities (node \#9). Although targeting the most central nodes does not necessarily lead to an effective change in the network (Fried et al., 2018), these findings suggest that a promising clinical strategy could be focusing on the individual's goals. In line with this, a recent review proposes that different types of goals may require substantially different types of clinical interventions in order to reduce hopelessness (Marchetti, Alloy, \& Koster, 2018). Hence, when targeting the individual's motivational structure, caution is recommended.

Third, the other most central item of the network tapped on the belief that in the future there will be more negative than positive times (node \#19), which was associated with lack of of faith and trust in the future (node \#15) (Nekanda-Trepka, Bishop, \& Blackburn, 1983). Interestingly, node \#19 was also linked with perceiving the future as vague and uncertain (node \#18). Moreover, mirroring this association, perceiving the future as dark (node \#7) was strictly related with the inability to imagine the future (node \#4) and viewing it as vague and uncertain (node \#18). Taken together, these findings suggest negative future expectations may be related to reduced capability to imagine specific and detailed future scenarios.

These pieces of evidence are of great interest, in that they could indicate a viable way to instill positive expectations about the future. Previous literature has shown that 
pessimism/hopelessness is strongly related to negative memory bias (Marchetti, Everaert, et al., 2018) and reduced capability to simulate one's own future is associated with diminished recall of specific personal memories (i.e., overgeneral autobiographical memory; Schacter, Addis, \& Buckner, 2008; Williams et al., 1996). Hence, it is possible to speculate that targeting biased memory processing could help improving future expectations. In line with this, preliminary evidence indicates the interventions targeting overgeneral autobiographical memory do lead to a significant reduction of hopelessness (Raes, Williams, \& Hermans, 2009; Serrano, Latorre, Gatz, \& Montanes, 2004).

Fourth, the analysis also revealed that both node centrality and predictability were negatively related to item mean levels (Beard et al., 2016). In other words, specific items may constitute the "backbones" of the hopelessness network, despite being less frequently agreed upon. In line with the network approach, this evidence underlines the need to focus not only on the mere intensity, but also on the specific role played by the each component of the network (Mullarkey et al., 2018). Future studies could explore whether the relationship between between centrality indexes and mean levels holds in the clinical population too, as previously reported (Beard et al., 2016).

Finally, it is worth mentioning that, on average, only about $36 \%$ of node variance could be explained by the neighboring nodes (i.e., predictability) and this implies that the majority of variance (i.e., 64\%) in the hopelessness components was not accounted for. In future studies, the focus could be broadened by including phenomena and mechanisms that are known to impact hopelessness, such as hope, cognitive style, loneliness, future orientation, overgeneral autobiographical memory, depressive symptoms, and suicidality (Abramson et al., 1989; Marchetti, Alloy, et al., 2018; Snyder, 2002).

This study is characterized by several strengths and limitations. First, the sample was large and highly representative of the general population, hence the reported findings are 
likely to be generalizable to the non-clinical population. Second, state-of-the-art network analyses were adopted to ensure the trustworthiness and replicability of the results. Third, the reported findings were stable, even after covariation of sociodemographic variables and items dichotomization. Fourth, by applying an innovative approach, this study proposed several testable hypotheses for future research.

Among the limitations of this study is possible to mention that, first, data were crosssectional in nature; hence, no directionality among items could be derived. However, this study generated a number of empirically testable hypotheses that future studies should pursue (Costantini \& Perugini, 2018). For instance, both ad-hoc manipulations and experience sampling studies could clarify the specific causal/temporal unfolding of the different components of hopelessness. Second, although being the current gold standard for measuring hopelessness, the BHS items are phrased in such a way that part of the causal flow is already present at intra-item level (i.e., "I might as well give up because I can't make things better for myself", underline added). Future studies could consider deconstructing such items in order to reach the optimal level of granularity for the network components (Borsboom, 2017). Third, given that this study was carried out in a community sample, the reported results cannot be extended to clinical samples. Future studies should complement these findings, by specifically investigating groups where hopelessness plays a major role, such as individuals with major depression, schizophrenia, or borderline personality disorder, etc.

In conclusion, by applying the network approach, this study represents an initial attempt to investigate the underlying structure of hopelessness. The analysis revelead that blocked goal-processing and negative expectations about the future are the most central nodes and, as such, potential loci of clinical interventions. This study also suggested that reducing the degree of vagueness and uncertainty of the anticipated future and increasing the 
imaginative skills about future events could be a viable way to reduce hopelessness and, eventually, improve mental health. 


\section{Compliance with Ethical Standards}

Conflict of Interest The author declares that he has no conflict of interest.

Ethical Approval All procedures performed in studies involving human participants were in accordance with the ethical standards of the institutional and/or national research committee and with the 1964 Helsinki declaration and its later amendments or comparable ethical standards.

Animal Rights Statement No animal studies were carried out by the authors for this article.

Informed Consent Informed consent was obtained from all individual participants included in the study. 


\section{Figure Caption}

Figure 1. Network model for the 20 items of the BHS. Positively worded items were reversed prior the network estimation.

Figure 2. Strength scores (centrality), shown as standardized $z$ scores.

Figure 3. Nonparametric bootstrapped difference test for strength. Gray boxes indicate no significant difference, whereas black boxes indicate statistically significant difference $(p<$ 0.05). Diagonal values represent the strength score of each node. 


\section{References}

Abramson, L. Y., Alloy, L. B., \& Metalsky, G. I. (1989). Hopelessness Depression - a Theory-Based Subtype of Depression. Psychological Review, 96(2), 358-372. doi: 10.1037/0033-295x.96.2.358

Beard, C., Millner, A. J., Forgeard, M. J., Fried, E. I., Hsu, K. J., Treadway, M. T., ... \& Björgvinsson, T. (2016). Network analysis of depression and anxiety symptom relationships in a psychiatric sample. Psychological medicine, 46, 3359-3369. doi: 10.1017/S0033291716002300

Beck, A. T., Steer, R. A., Beck, J. S., \& Newman, C. F. (1993). Hopelessness, Depression, Suicidal Ideation, and Clinical-Diagnosis of Depression. Suicide and Life-Threatening Behavior, 23(2), 139-145. doi: 10.1111/j.1943-278X.1993.tb00378.x

Beck, A. T., Weissman, A., Lester, D., \& Trexler, L. (1974). The measurement of pessimism: the hopelessness scale. Journal of Consulting and Clinical Psychology, 42(6), 861865. doi: $10.1037 / \mathrm{h} 0037562$

Borsboom, D. (2017). A network theory of mental disorders. World psychiatry, 16, 5-13. doi: $10.1002 /$ wps. 20375

Borsboom, D., \& Cramer, A. O. J. (2013). Network Analysis: An Integrative Approach to the Structure of Psychopathology. Annual Review of Clinical Psychology, 9, 91-121. doi:10.1146/annurev-clinpsy-050212-185608

Borsboom, D., Fried, E. I., Epskamp, S., Waldorp, L. J., van Borkulo, C. D., van der Maas, H. L. J., \& Cramer, A. O. J. (2017). False Alarm? A Comprehensive Reanalysis of "Evidence That Psychopathology Symptom Networks Have Limited Replicability" by Forbes, Wright, Markon, and Krueger (2017). Journal of Abnormal Psychology, 126(7), 989-999. doi:10.1037/abn0000306

Briganti, G., Kempenaers, C., Braun, S., Fried, E. I., \& Linkowski, P. (2018). Network analysis of empathy items from the interpersonal reactivity index in 1973 young adults. Psychiatry Research, 265, 87-92. doi: 10.1016/j.psychres.2018.03.082

Costantini, G., \& Perugini, M. (2018). A Framework for Testing Causality in Personality Research. European Journal of Personality, 32, 254-268. doi:10.1002/per.2150

Crawford, T., \& Ellis, A. J. (1989). A dictionary of rational-emotive feelings and behaviors. Journal of Rational-Emotive \& Cognitive-Behavior Therapy, 7(1), 3-28. doi: $10.1007 / \mathrm{bf02175569}$

Dalege, J., Borsboom, D., van Harreveld, F., van den Berg, H., Conner, M., \& van der Maas, H. L. J. (2016). Toward a Formalized Account of Attitudes: The Causal Attitude Network (CAN) Model. Psychological Review, 123(1), 2-22. doi:10.1037/a0039802

Dalege, J., Borsboom, D., van Harreveld, F., \& van der Maas, H. L. J. (2017). Network Analysis on Attitudes: A Brief Tutorial. Social Psychological and Personality Science, 8(5), 528-537. doi:10.1177/1948550617709827

Epskamp, S., Borsboom, D., \& Fried, E. I. (2018). Estimating psychological networks and their accuracy: A tutorial paper. Behavior Research Methods, 50(1), 195-212. doi:10.3758/s13428-017-0862-1

Epskamp, S., Cramer, A. O. J., Waldorp, L. J., Schmittmann, V. D., \& Borsboom, D. (2012). qgraph: Network Visualizations of Relationships in Psychometric Data. Journal of Statistical Software, 48(4), 1-18. doi: 10.18637/jss.v048.i04

Epskamp, S., \& Fried, E. I. (2018). A tutorial on regularized partial correlation networks. Psychological Methods. doi:10.1037/met0000167

Fischer, R., \& Chalmers, A. (2008). Is optimism universal? A meta-analytical investigation of optimism levels across 22 nations. Personality and Individual Differences, 45, 378382. doi: $10.1016 /$ j.paid.2008.05.008 
Franklin, J. C., Ribeiro, J. D., Fox, K. R., Bentley, K. H., Kleiman, E. M., Huang, X. Y. N., . . . Nock, M. K. (2017). Risk Factors for Suicidal Thoughts and Behaviors: A MetaAnalysis of 50 Years of Research. Psychological Bulletin, 143(2), 187-U121. doi:10.1037/bul0000084

Greene, S. M. (1981). Levels of Measured Hopelessness in the General-Population. British Journal of Clinical Psychology, 20(Feb), 11-14. doi: 10.1111/j.20448260.1981.tb00490.x

Haatainen, K. M., Tanskanen, A., Kylma, J., Antikainen, R., Hintikka, J., Honkalampi, K., . . . Viinamaki, H. (2003). Life events are important in the course of hopelessness - a 2year follow-up study in a general population. Social Psychiatry and Psychiatric Epidemiology, 38(8), 436-441. doi:10.1007/s00127-003-0660-1

Haatainen, K. M., Tanskanen, A., Kylma, J., Honkalampi, K., Koivumaa-Honkanen, H., Hintikka, J., . . Viinamaki, H. (2003). Stable hopelessness and its predictors in a general population: A 2-year follow-up study. Suicide and Life-Threatening Behavior, 33(4), 373-380. doi: 10.1521/suli.33.4.373.25237

Hadley, S. A., \& MacLeod, A. K. (2010). Conditional goal-setting, personal goals and hopelessness about the future. Cognition \& Emotion, 24(7), 1191-1198. doi: 10.1080/02699930903122521

Hanna, D., White, R., Lyons, K., McParland, M. J., Shannon, C., \& Mulholland, C. (2011). The structure of the Beck Hopelessness Scale: A confirmatory factor analysis in UK students. Personality and Individual Differences, 51, 17-22. doi: 10.1016/j.paid.2011.03.001

Haslbeck, J. M. B., \& Waldorp, L. J. (2018). How well do network models predict observations? On the importance of predictability in network models. Behavior Research Methods, 50(2), 853-861. doi:10.3758/s13428-017-0910-x

Iliceto, P., \& Fino, E. (2015). Beck Hopelessness Scale (BHS) A Second-Order Confirmatory Factor Analysis. European Journal of Psychological Assessment, 31(1), 31-37. doi:10.1027/1015-5759/a000201

Jones, P. J. (2018). networktools: Assorted Tools for Identifying Important Nodes in Networks. R package version 1.2.0. https://CRAN.Rproject.org/package $=$ networktools

Krampen, G. (1994). Skalen zur Erfassung von Hoffnungslosigkeit (H-Skalen). Deutsche Bearbeitung und Weiterentwicklung der H-Skala von Aaron T. Beck. Göttingen: Hogrefe.

Krampen, G. (2004). Hopelessness Scales. Primary data from the standardization sample. [Translated Title] (Version 1) [Files on CD-ROM]. Trier: Center for Research Data in Psychology: PsychData of the Leibniz Institute for Psychology Information ZPID. https://doi.org/10.5160/psychdata.kngr90sk04

Lysaker, P. H., Davis, L. W., \& Hunter, N. L. (2004). Neurocognitive, social and clinical correlates of two domains of hopelessness in schizophrenia. Schizophrenia Research, 70(2-3), 277-285. doi:10.1016/j.schres.2004.01.007

Mac Giollabhui, N., Hamilton, J. L., Nielsen, J., Connolly, S. L., Stange, J. P., Varga, S., . . . Alloy, L. B. (2018). Negative Cognitive Style Interacts With Negative Life Events to Predict First Onset of a Major Depressive Episode in Adolescence via Hopelessness. Journal of Abnormal Psychology, 127(1), 1-11. doi:10.1037/abn0000301

Marchetti, I., Alloy, L. B., \& Koster, E. H. W. (2018). Breaking the vise of hopelessness: Targeting its antecedents, components, and context. Manuscript in preparation.

Marchetti, I., Everaert, J., Dainer-Best, J., Loeys, T., Beevers, C. G., \& Koster, E. H. W. (2018). Specificity and overlap of emotional biases in depression. Journal of Affective Disorders, 225, 404-412. doi: 10.1016/j.jad.2017.08.037 
Marchetti, I., Loeys, T., Alloy, L. B., \& Koster, E. H. W. (2016). Unveiling the structure of cognitive vulnerability for depression: Specificity and overlap. PLoS One. doi: 10.1371/journal.pone.0168612.

Marshall, G. N., Wortman, C. B., Kusulas, J. W., Hervig, L. K., \& Vickers, R. R. (1992). Distinguishing Optimism from Pessimism - Relations to Fundamental Dimensions of Mood and Personality. Journal of Personality and Social Psychology, 62(6), 10671074. doi: 10.1037//0022-3514.62.6.1067

Melges, F. T., \& Bowlby, J. (1969). Types of Hopelessness in Psychopathological Process. Archives of General Psychiatry, 20(6), 690-699. doi: 10.1001/archpsyc.1969.01740180074007

Mullarkey, M., Marchetti, I., \& Beevers, C. G. (2018). Using a Network Analysis to Identify Central Symptoms of Adolescent Depression. Journal of Clinical Child and Adolescent Clinical Psychology. doi:10.1080/15374416.2018.1437735

Nekanda-Trepka, C. J. S., Bishop, S., \& Blackburn, I. M. (1983). Hopelessness and depression. British Journal of Clinical Psychology, 22, 49-60. doi: 10.1111/j.20448260.1983.tb00578.x

Peterson, C. (2000). The future of optimism. American Psychologist, 55(1), 44-55. doi: 10.1037//0003-066x.55.1.44

Raes, F., Williams, J. M. G., \& Hermans, D. (2009). Reducing cognitive vulnerability to depression: A preliminary investigation of MEmory Specificity Training (MEST) in inpatients with depressive symptomatology. Journal of Behavior Therapy and Experimental Psychiatry, 40(1), 24-38. doi:10.1016/j.jbtep.2008.03.001

Ratcliffe, M. (2015). Experiences of Depression: A Study in Phenomenology. Oxford: Oxford University Press.

Roepke, A. M., \& Seligman, M. E. (2016). Depression and prospection. British Journal of Clinical Psychology, 55(1), 23-48. doi:10.1111/bjc.12087

Schacter, D. L., Addis, D. R., \& Buckner, R. L. (2008). Episodic simulation of future events Concepts, data, and applications. Year in Cognitive Neuroscience 2008, 1124, 39-60. doi:10.1196/annals.1440.001

Schmittmann, V. D., Cramer, A. O., Waldorp, L. J., Epskamp, S., Kievit, R. A., \& Borsboom, D. (2013). Deconstructing the construct: A network perspective on psychological phenomena. New ideas in psychology, 31, 43-53. doi: 10.1016/j.newideapsych.2011.02.007

Seligman, M. E. P. (1975). Helplessness: On depression, development, and death. San Francisco: Freeman.

Serrano, J. P., Latorre, J. M., Gatz, M., \& Montanes, J. (2004). Life review therapy using autobiographical retrieval practice for older adults with depressive symptomatology. Psychology and Aging, 19(2), 272-277. doi:10.1037/0882-7974.19.2.272

Snyder, C. R. (2002). Hope theory: Rainbows in the mind. Psychological Inquiry, 13(4), 249275. doi: 10.1207/S15327965PLI1304_01

Steed, L. (2001). Further validity and reliability evidence for Beck Hopelessness Scale scores in a nonclinical sample. Educational and Psychological Measurement, 61(2), 303-316. doi: 10.1177/00131640121971121

Valente, T. W. (2012). Network Interventions. Science, 337(6090), 49-53. doi: $10.1126 /$ science. 1217330

Van Borkulo, C. D., Borsboom, D., Epskamp, S., Blanken, T. F., Boschloo, L., Schoevers, R. A., \& Waldorp, L. J. (2014). A new method for constructing networks from binary data. Scientific reports, 4, 5918. doi: 10.1038/srep05918 
Williams, J. M. G., Ellis, N. C., Tyers, C., Healy, H., Rose, G., \& MacLeod, A. K. (1996). The specificity of autobiographical memory and imageability of the future. Memory \& Cognition, 24(1), 116-125. doi: 10.3758/Bf03197278

Zhang, W. C., Jia, C. X., Hu, X., Qiu, H. M., \& Liu, X. C. (2015). Beck hopelessness scale: psychometric properties among rural Chinese suicide attempters and nonattempters. Death studies, 39, 442-446. doi: 10.1080/07481187.2014.970300 


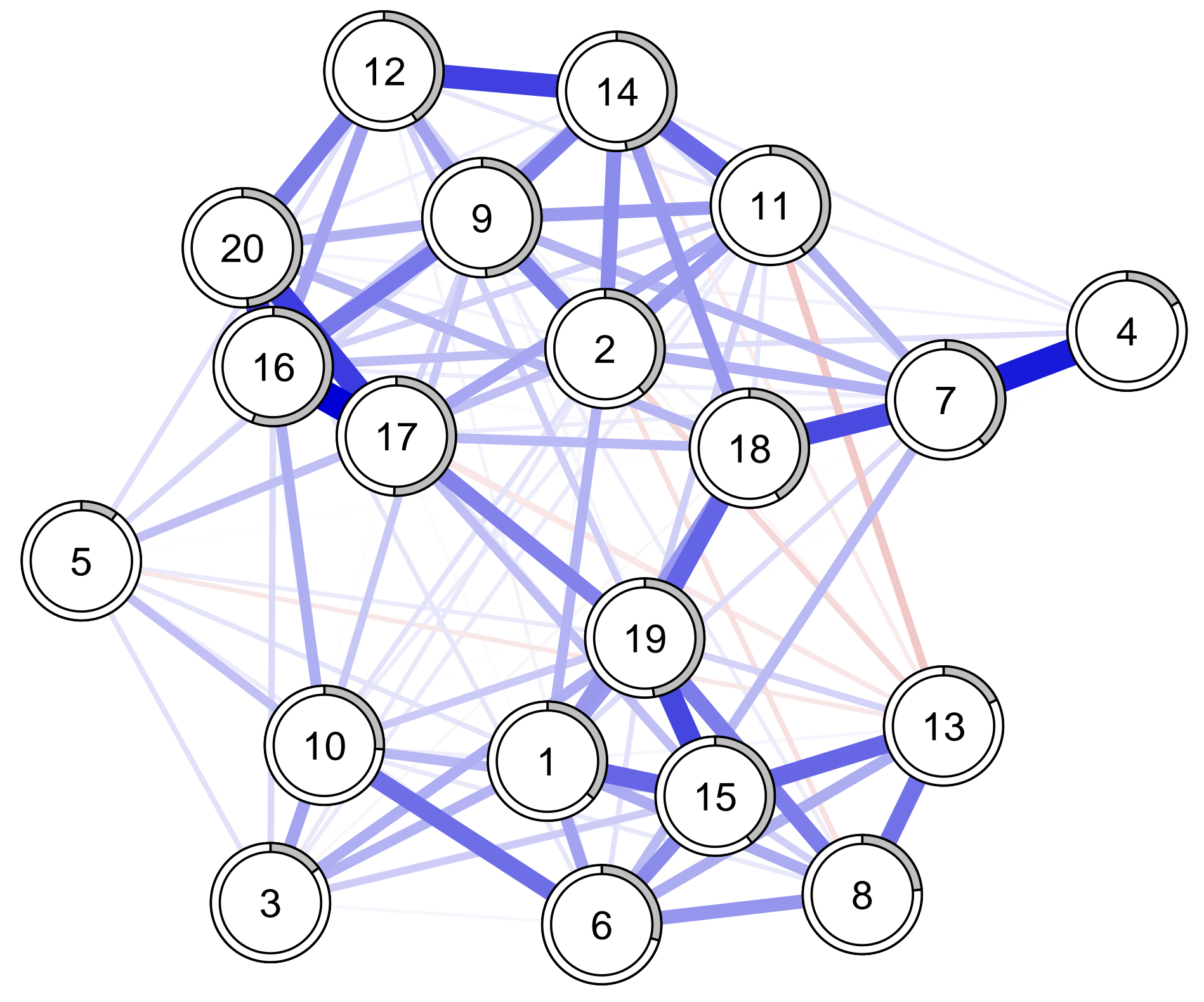

1: [NOT] hope-and-enthusiasm

2: giving-up/helplessness

3: [NOT] up-feeling

4: not-able-to-imagine-future

5: [NOT] enough-time

6: [NOT] expect-to-succeed

7: dark-future

8: [NOT] particularly-lucky

9: can't-get-the-breaks

10: [NOT] preparation-for-future

11: unpleasantness-ahead

12: not-getting

13: [NOT] happier-future

14: not-working-out

15: [NOT] faith-in-the-future

16: giving-up-on-wanting

17: no-future-satisfication

18: uncertain-future

19: [NOT] better-future

20: not-trying 
19.[NOT] better-future -

16.giving-up-on-wanting -

9.can't-get-the-breaks -

17.no-future-satisfication -

14.not-working-out -

15.[NOT] faith-in-the-future -

2.giving-up/helplessness -

7.dark-future -

1.[NOT] hope-and-enthusiasm -

11.unpleasantness-ahead -

20.not-trying -

18.uncertain-future -

6.[NOT] expect-to-succeed -

12.not-getting -

10.[NOT] preparation-for-future -

13.[NOT] happier-future -

8.[NOT] particularly-lucky -

3.[NOT] up-feeling -

5.[NOT] enough-time -

4. not-able-to-imagine-future -

$\begin{array}{llll}1 & -1 & 1 & 1\end{array}$




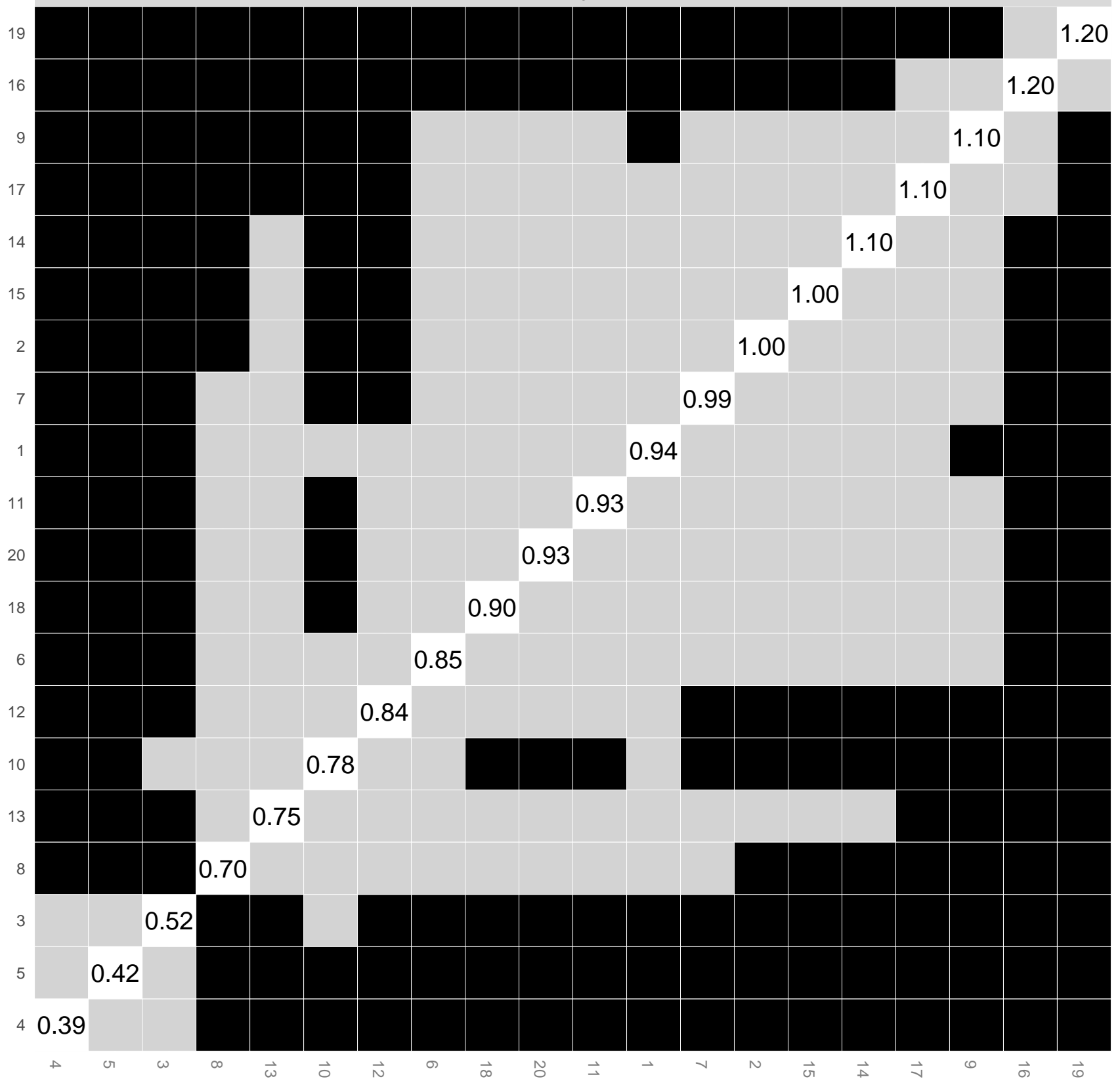




\section{Supplementary Material}

Table S1. Means, standard deviations, skewness, and kurtosis $(\mathrm{n}=1985)$

\begin{tabular}{|c|c|c|c|c|}
\hline Item & $M$ & $S D$ & Skewness & Kurtosis \\
\hline 1. [NOT] hope-and-enthusiasm & 2.65 & 1.19 & 0.62 & 0.12 \\
\hline 2. giving-up/helplessness & 2.56 & 1.38 & 0.65 & -0.44 \\
\hline 3. [NOT] up-feeling & 2.57 & 1.22 & 0.75 & 0.34 \\
\hline 4. not-able-to-imagine-future & 3.64 & 1.49 & -0.08 & -0.95 \\
\hline 5. [NOT] enough-time & 2.69 & 1.29 & 0.67 & -0.04 \\
\hline 6. [NOT] expect-to-succeed & 2.93 & 1.30 & 0.44 & -0.27 \\
\hline 7. dark-future & 2.96 & 1.41 & 0.36 & -0.68 \\
\hline 8. [NOT] particularly-lucky & 3.34 & 1.36 & 0.24 & -0.58 \\
\hline 9. can't-get-the-breaks & 2.28 & 1.23 & 0.88 & 0.28 \\
\hline 10. [NOT] preparation-for-future & 2.63 & 1.14 & 0.60 & 0.36 \\
\hline 11. unpleasantness-ahead & 2.54 & 1.30 & 0.62 & -0.33 \\
\hline 12. not-getting & 2.82 & 1.44 & 0.46 & -0.73 \\
\hline 13. [NOT] happier-future & 3.61 & 1.29 & 0.11 & -0.58 \\
\hline 14. not-working-out & 2.80 & 1.30 & 0.49 & -0.36 \\
\hline 15. [NOT] faith-in-the-future & 2.64 & 1.20 & 0.64 & 0.20 \\
\hline 16. giving-up-on-wanting & 2.23 & 1.25 & 0.94 & 0.37 \\
\hline 17. no-future-satisfication & 2.26 & 1.31 & 0.87 & -0.07 \\
\hline 18. uncertain-future & 2.94 & 1.33 & 0.29 & -0.62 \\
\hline 19. [NOT] better-future & 2.65 & 1.14 & 0.50 & 0.06 \\
\hline 20. not-trying & 2.47 & 1.31 & 0.63 & -0.38 \\
\hline
\end{tabular}

Note: [NOT] indicates already reversed items. Higher mean levels indicate higher levels of hopelessness across all the items. 
Table S2. Zero-order polychoric correlations among the BHS items

\begin{tabular}{|c|c|c|c|c|c|c|c|c|c|c|c|c|c|c|c|c|c|c|c|c|}
\hline items & 1 & 2 & 3 & 4 & 5 & 6 & 7 & 8 & 9 & 10 & 11 & 12 & 13 & 14 & 15 & 16 & 17 & 18 & 19 & 20 \\
\hline 1 & 1 & 0.35 & 0.31 & 0.16 & 0.21 & 0.4 & 0.35 & 0.34 & 0.33 & 0.33 & 0.32 & 0.31 & 0.18 & 0.34 & 0.49 & 0.33 & 0.34 & 0.43 & 0.49 & 0.34 \\
\hline 2 & 0.35 & 1 & 0.25 & 0.25 & 0.27 & 0.24 & 0.44 & 0.08 & 0.55 & 32 & 49 & & -0.07 & 53 & .23 & 0.51 & 0.44 & 39 & 35 & 0.44 \\
\hline 3 & 0.31 & 0.25 & 1 & 0.05 & 0.18 & 0.23 & 0.15 & 0.17 & 0.26 & 0.31 & 0.25 & 0.17 & 0.05 & 0.22 & 0.3 & 0.3 & 0.28 & 0.17 & 0.35 & 0.25 \\
\hline 4 & 0.16 & 0.25 & 0.05 & 1 & 0.04 & 0.12 & 0.42 & 0.1 & 0.24 & 0.14 & 0.25 & 0.19 & 0.04 & 0.25 & 0.09 & 0.18 & 0.19 & 0.25 & 0.13 & 0.22 \\
\hline 5 & 0.21 & 0.27 & 0.18 & 0.04 & 1 & 0.18 & 0.2 & 0.12 & 0.27 & 0.24 & 0.21 & 0.24 & -0.04 & 0.25 & 0.15 & 0.21 & 0.21 & 0.19 & 0.22 & 0.15 \\
\hline 6 & 0.4 & 0.24 & 0.23 & 0.12 & 0.18 & 1 & 0.31 & 0.35 & 0.28 & 0.39 & 0.19 & 0.27 & 0.25 & 0.26 & 0.43 & 0.29 & 0.32 & 0.24 & 0.39 & 0.3 \\
\hline 7 & 0.35 & 0.44 & 0.15 & 0.42 & 0.2 & 0.31 & 1 & 0.18 & 0.47 & 0.29 & 0.45 & 0.36 & 0.06 & 0.44 & 0.29 & 0.44 & 0.42 & 0.51 & 0.35 & 0.38 \\
\hline 8 & .34 & 0.08 & 0.17 & 0.1 & 0.12 & 0.35 & 0.18 & 1 & 0.2 & 0.25 & 0.11 & 0.2 & 0.3 & 0.16 & 0.36 & 0.15 & 0.17 & 0.18 & 0.4 & 0.16 \\
\hline 9 & 0.33 & 0.55 & 0.26 & 0.24 & 0.27 & 0.28 & 0.47 & 0.2 & 1 & 0.38 & 0.55 & 0.54 & -0.03 & 0.59 & .33 & 63 & .55 & 0.45 & .43 & 0.56 \\
\hline 10 & 0.33 & 0.32 & 0.31 & 0.14 & 0.24 & 0.39 & 0.29 & 0.25 & 0.38 & 1 & 0.32 & 0.3 & 0.1 & 0.29 & 0.38 & 0.41 & 0.36 & 0.25 & 0.4 & 0.35 \\
\hline 11 & 0.32 & 0.49 & 0.25 & 0.25 & 0.21 & 0.19 & 0.45 & 0.11 & 0.55 & 0.32 & 1 & 0.45 & -0.07 & 0.56 & 0.27 & 0.53 & 0.51 & 0.43 & 0.41 & 0.46 \\
\hline 12 & 0.31 & 0.45 & 0.17 & 0.19 & 0.24 & 0.27 & 0.36 & 0.2 & 0.54 & 0.3 & 0.45 & 1 & -0.03 & 0.57 & 0.28 & 0.56 & 0.48 & 0.39 & 4 & 0.54 \\
\hline 13 & 0.18 & -0.07 & 0.05 & 0.04 & -0.04 & 0.25 & 0.06 & 0.3 & -0.03 & 0.1 & -0.07 & -0.03 & 1 & -0.05 & 0.32 & -0.04 & 0 & 0.07 & 0.22 & 0.05 \\
\hline 14 & & 0.53 & 0.22 & 0.25 & 0.25 & & 0.44 & & & & & & -0.05 & 1 & 0.27 & 0.56 & 0.48 & & & 0.5 \\
\hline 15 & 49 & 0.23 & 0.3 & 0.09 & 0.15 & 0.43 & 0.29 & 0.36 & 0.33 & 0.38 & 0.27 & 0.28 & 0.32 & 0.27 & 1 & 0.36 & 0.41 & 0.36 & 0.55 & 0.35 \\
\hline 16 & 0.33 & 0.51 & 0.3 & 0.18 & 0.21 & 0.29 & 0.44 & 0.15 & 0.63 & 0.41 & 0.53 & 0.56 & -0.04 & 0.56 & 0.36 & 1 & 0.69 & 0.47 & 0.46 & 0.66 \\
\hline 17 & .34 & 0.44 & 0.28 & 0.19 & 0.21 & 0.32 & 0.42 & 0.17 & 0.55 & 0.36 & 0.51 & 0.48 & 0 & 0.48 & 0.41 & 0.69 & 1 & 0.5 & 0.53 & 0.64 \\
\hline 18 & 0.43 & 0.39 & 0.17 & 0.25 & 0.19 & 0.24 & 0.51 & 0.18 & 0.45 & 0.25 & 0.43 & 0.39 & 0.07 & 0.48 & 0.36 & 0.47 & 0.5 & 1 & 0.52 & 0.47 \\
\hline 19 & 0.49 & 0.35 & 0.35 & 0.13 & 0.22 & 0.39 & 0.35 & 0.4 & 0.43 & 0.4 & 0.41 & 0.4 & 0.22 & 0.4 & 0.55 & 0.46 & 0.53 & 0.52 & 1 & 0.43 \\
\hline 20 & 0.34 & 0.44 & 0.25 & 0.22 & 0.15 & 0.3 & 0.38 & 0.16 & 0.56 & 0.35 & 0.46 & 0.54 & 0.05 & 0.5 & 0.35 & 0.66 & 0.64 & 0.47 & 0.43 & \\
\hline
\end{tabular}

Note: positively worded items (i.e., 1, 3, 5, 6, 8, 10, 13, 15, 19) were already reversed prior the estimation of the polychoric correlations. Across all items, higher values indicate higher levels of hopelessness 
Figure S1. Nonparametric bootstrapped confidence intervals of estimated edges. The red line represents the estimated edge, while the dark area indicates the $95 \%$ bootstrap confidence interval.

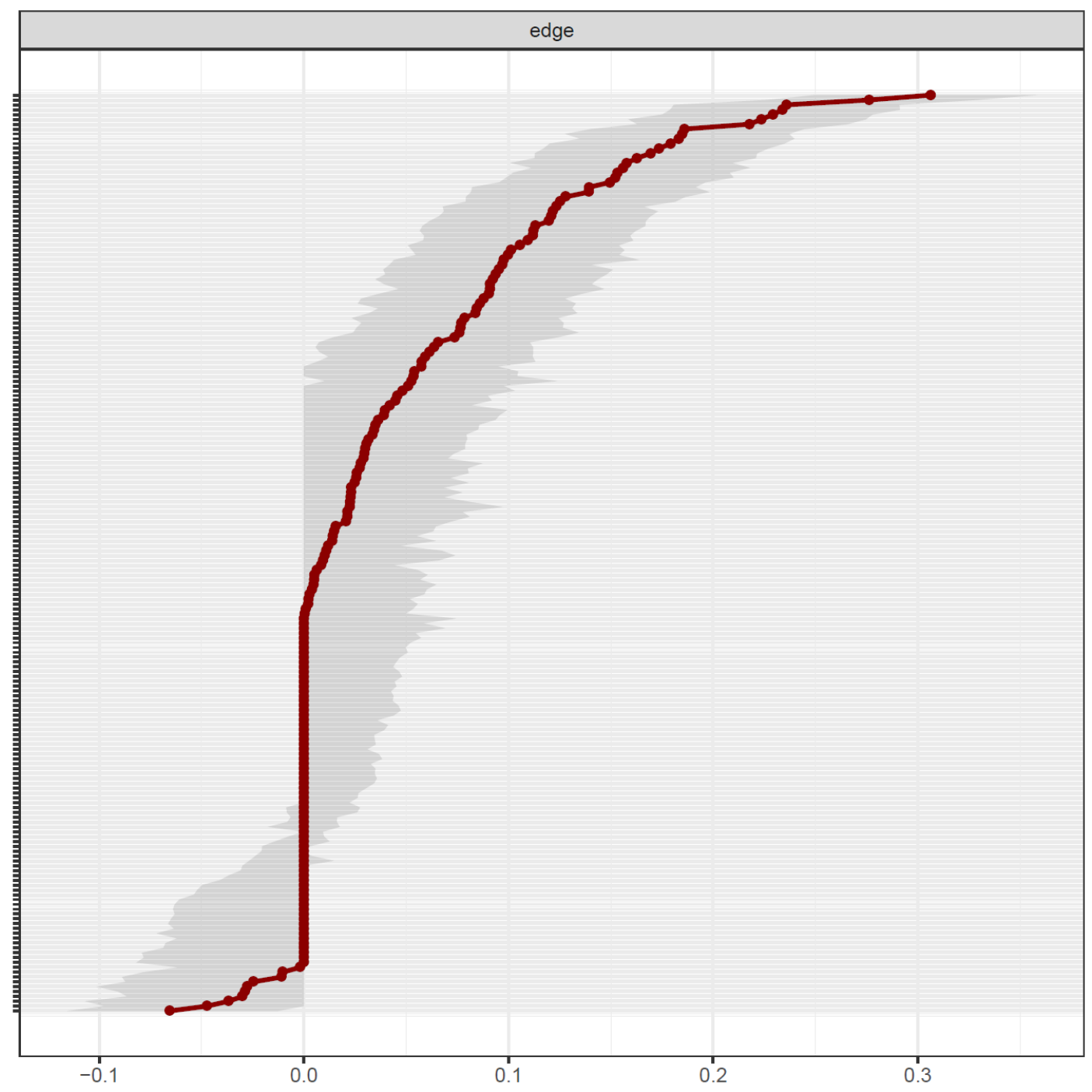


Figure S2. Nonparametric bootstrapped difference test for edges. Gray boxes indicate no significant difference, whereas black boxes indicate statistically significant difference $(p<$ 0.05). Diagonal color and saturation represent the magnitude and direction of each estimated edge.

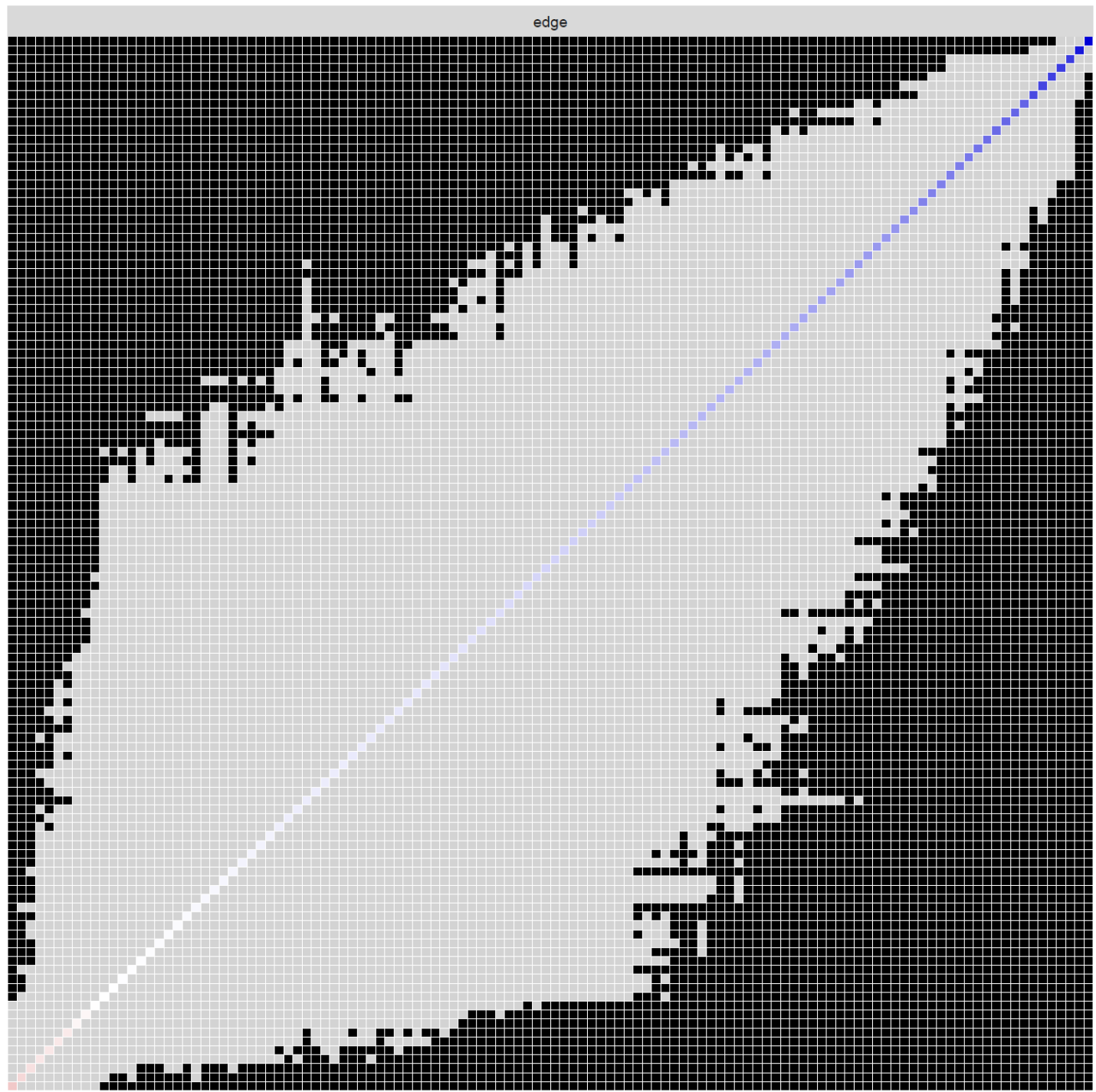


Figure S3. Visual representation of the original network estimated and the same network, after covariating for age, gender, and employment status.

original network

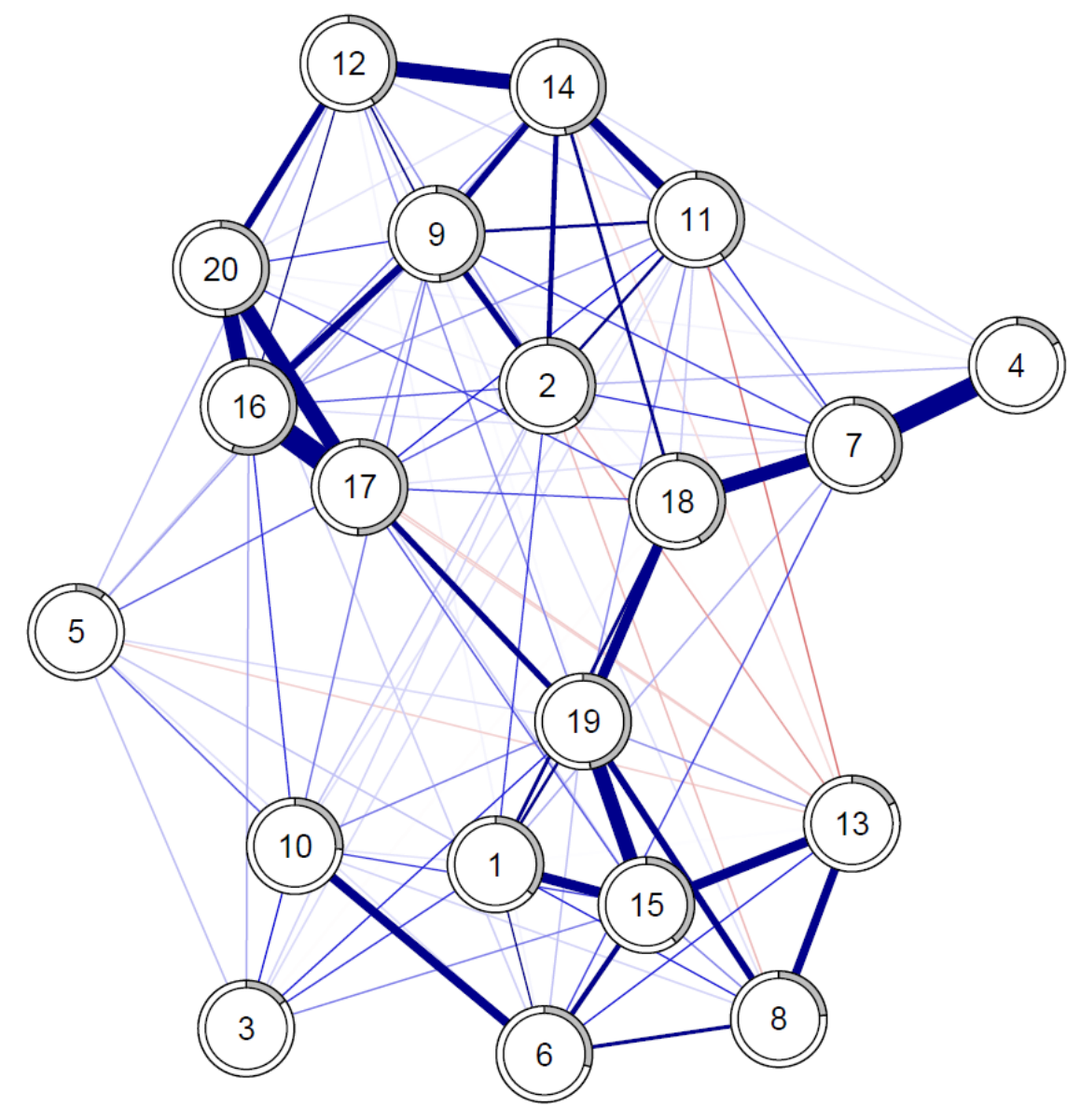

network after covariating age, gender, and employment

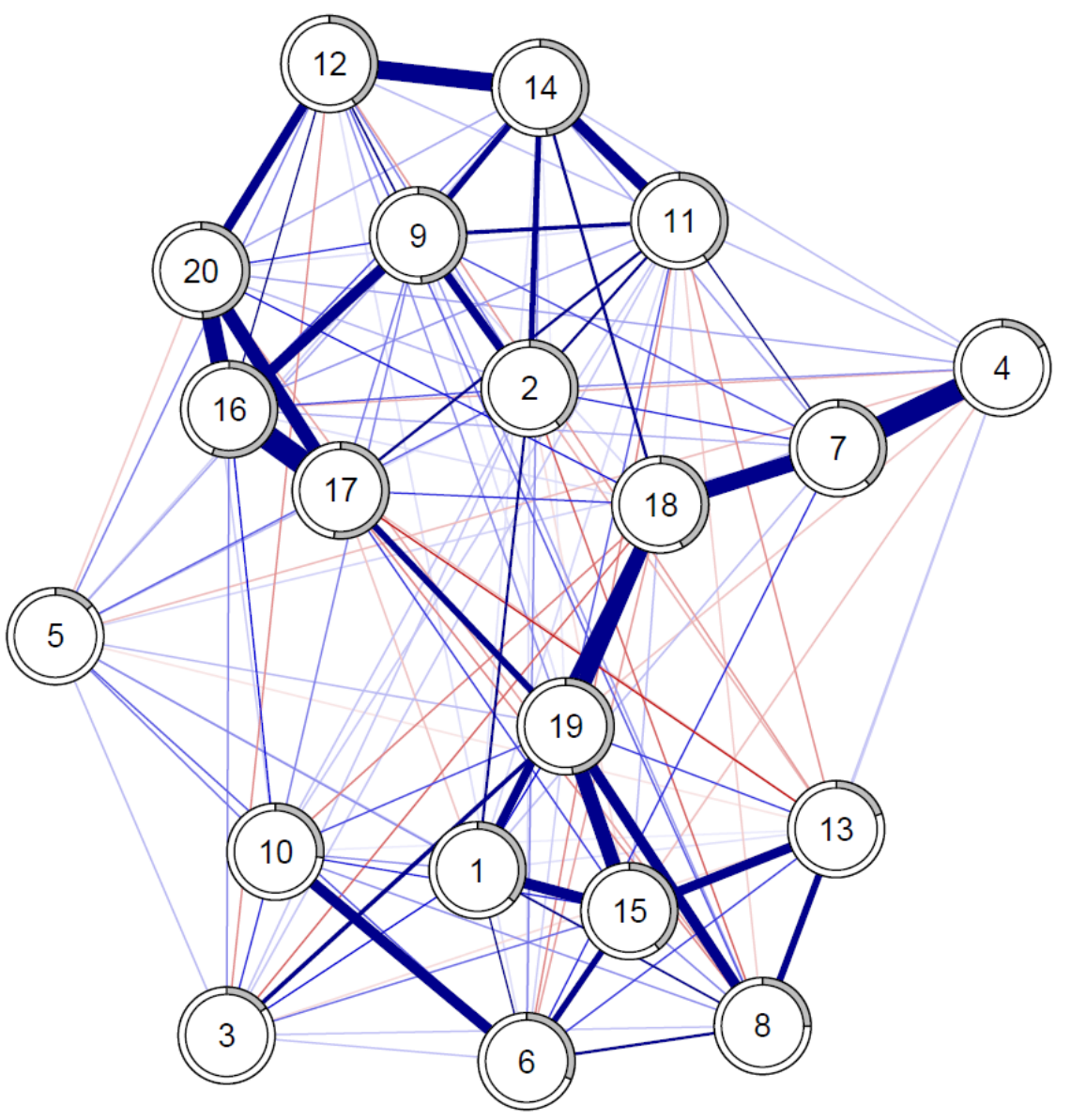


Figure S4. Visual representation of the original network estimated on 6-point Likert scale items (i.e.. EBIC graphical LASSO network) and the network estimated on dichotomized items (i.e., Ising network).

\section{6-point Likert}

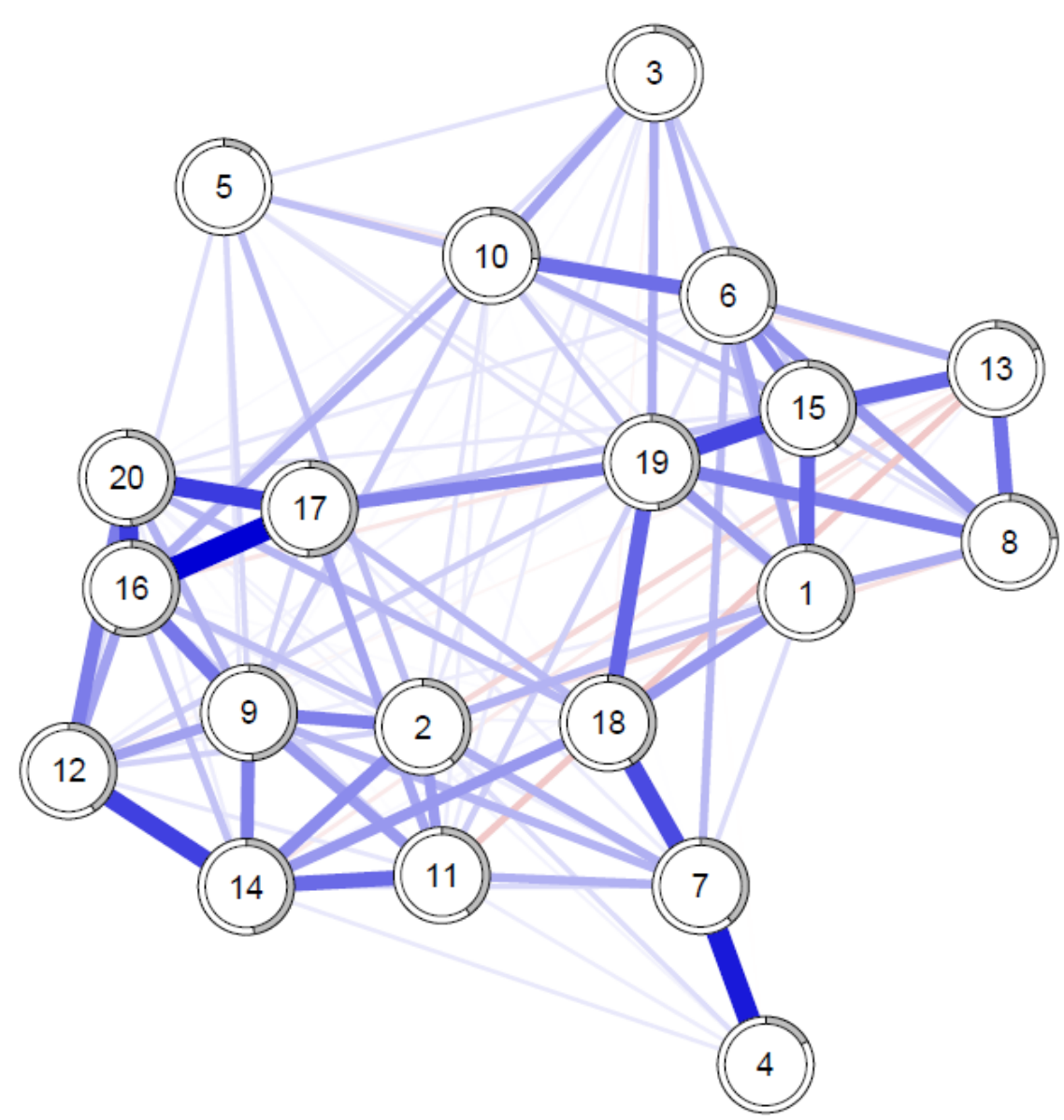

\section{binarized items}

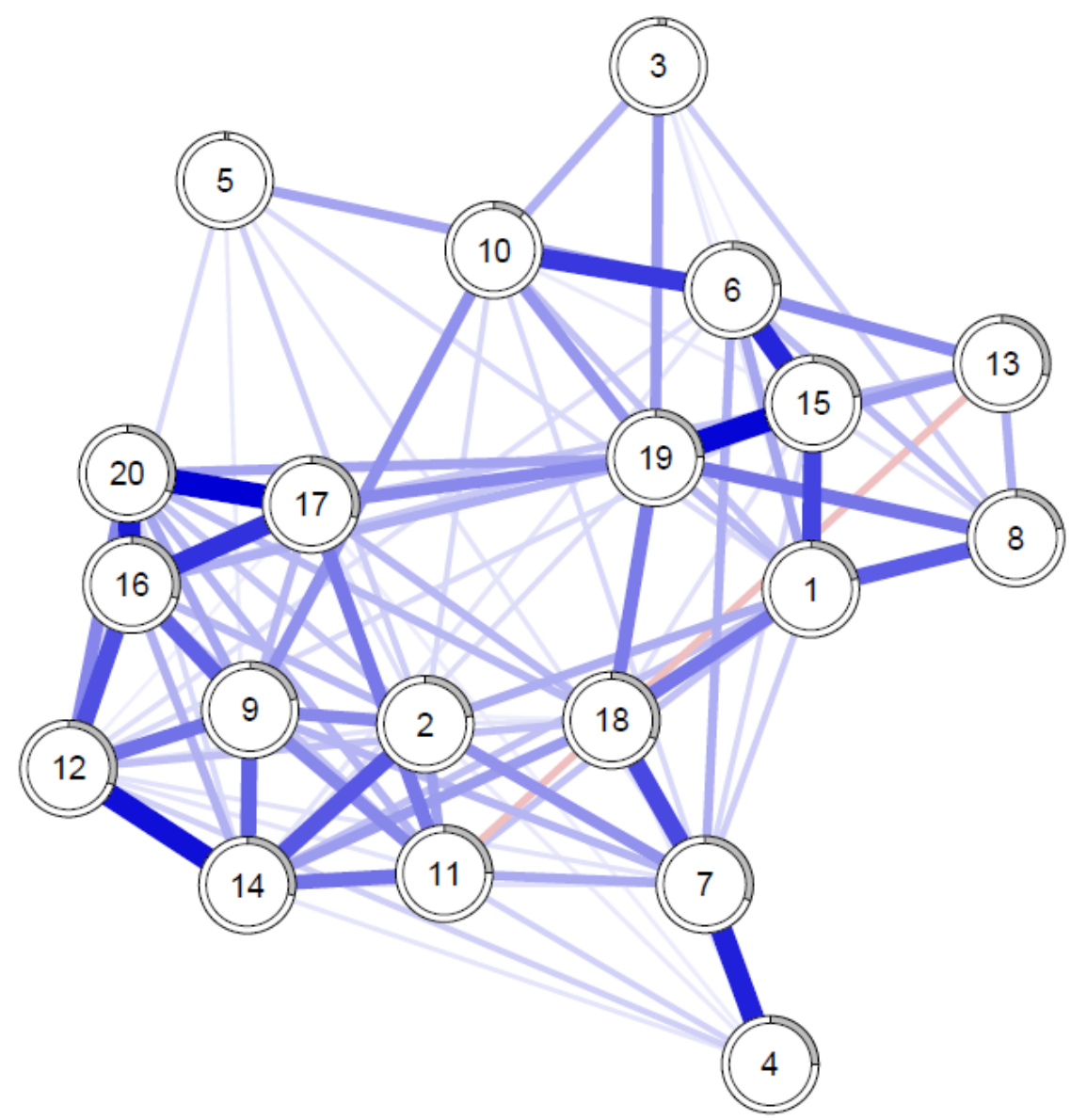

